$\underline{\mathbf{P}-80}$

\title{
Subcritical Water Extraction (SWE) of Anthocyanin from Arabica Coffee Pulp
}

\author{
Niramol Punbusayakul ${ }^{2,}$, Norashikin Saim ${ }^{1}$, Zaibunnisa Abdul Haiyee ${ }^{1}$, Warin Sarnkhuankaew ${ }^{2}$ \\ and Rozita Osman ${ }^{1}$ \\ ${ }^{l}$ Faculty of Applied Sciences, Universiti Teknologi MARA, 40450 Shah Alam, Malaysia; ${ }^{2}$ School of Agro-Industry Mae \\ Fah Luang University, 57100 Chiang Rai, Thailand; E-mail: pniramol2@gmail.com
}

Coffee pulp which is $45-55 \%$ of coffee cherry is the main by-product from coffee processing industry. Analysis of cyanidin-3rutinoside (C-3-R), the major anthocyanin in coffee pulp is important for the development of value-added products. The SWE parameters including extraction temperature, extraction time and cycle were studied for selective and efficient extraction of C3-R from Arabica coffee pulp. Quantitative analysis was obtained using high performance liquid chromatography (HPLC). SWE is a promising green approach in the extraction of bioactive compounds from plants materials.

Keywords: Anthocyanin, Arabica coffee, coffee pulp, subcritical water extraction. 